\title{
EVALUATION AND COMPARISON OF A NEW TYPE OF TEMPORARY IMMERSION SYSTEM (TIS) BIOREACTORS FOR MYRTLE (MYRTUS COMMUNIS L.)
}

\author{
AKA KAÇAR, Y. $.^{1,2^{*}}-$ BIÇEN, B. $^{2}-$ ŞIMŞEK, Ö. ${ }^{3}-$ DÖNMEZ, D. ${ }^{2}-$ EROL, M. H. ${ }^{2}$ \\ ${ }^{1}$ Horticulture Department, Agriculture Faculty, Çukurova University, Adana 01330, Turkey \\ ${ }^{2}$ Biotechnology Research and Application Center, Çukurova University, Adana 01330, Turkey \\ ${ }^{3}$ Horticulture Department, Agriculture Faculty, Erciyes University, Kayseri 38280, Turkey \\ *Corresponding author \\ e-mail: ykacar@cu.edu.tr; phone: +90-322-338-6084(2146)
}

(Received $4^{\text {th }}$ Oct 2019; accepted $4^{\text {th }}$ Dec 2019)

\begin{abstract}
Myrtle is known to be an important aromatic and medicinal plant species. Myrtle plant is consumed mainly as spice having several uses in the perfumery, food and pharmaceutical industries. It is also widely used for ornamental purposes such as fencing, roadsides, and green cutting. Plant tissue culture techniques are an essential tool to micropropagate for the plant producer. TIS (Temporary immersion systems) is an alternative plant tissue culture technique for micropropagation mechanism in plant species. A new culture vessel named PlantForm TIS bioreactor has been recently developed. In the present study, we aimed to evaluate and compare a new type of TIS bioreactors with agar media for myrtle. MS (Murashige and Skoog) medium supplemented with $1 \mathrm{mg} \mathrm{l}^{-1}$ BAP (benzylaminopurine) was used for multiplication, with $1 \mathrm{mg} \mathrm{l}^{-1}$ IBA (indole-3-butyric acid) for rooting. Two different immersion times $(4 \mathrm{~h}$ and $8 \mathrm{~h}$ ) were compared with the solid agar medium. Plantlets growing in PlantForm systems performed better than those in the solid medium. All data recorded at micropropagation and rooting treatments were much more successful. In conclusion, PlantForm TIS bioreactor presents a valid alternative to traditional in vitro micropropagation and rooting systems, resulting in a reduction of cost, labor, and time for the mass propagation.
\end{abstract}

Keywords: benzylaminopurine, micropropagation, PlantForm, plant tissue culture, bioreactor

\section{Introduction}

Myrtus communis L., commonly named myrtle, a valuable medicinal and aromatic species (Wannes et al., 2010; Dahmoune et al., 2015). Myrtle is known to be a member of the Myrtaceae family, which contains 3,000 species naturally growing in tropical and subtropical regions. Myrtle is mostly consumed as spice having several practices in the food, pharmaceutical, and industries (Sumbul et al., 2011; Casaburi et al., 2015). Myrtle plant could also be used as ornamental purposes such as fencing, roadsides, and green cutting (Shekafandeh, 2007; Danial, 2009). Myrtle broadly grows in 500-600 m altitude, most notably in pine forests and riversides in the Taurus Mountains of Turkey (Aydin and Özcan, 2007). Myrtle has several genotypes with yellowish-white or bluish-black colored fruits (Şan et al., 2015).

Many different biotechnological methods could be performed to fruit crops to get better ones during plant breeding strategies. One of the critical applications of biotechnology is known to be plant tissue culture in fruit science. Plant tissue culture techniques include several processes of clonal propagation and micropropagation. Known technologies in plant biotechnology have been expanded to contain virus elimination, somatic embryogenesis, organogenesis, production of haploid plants and 
somatic hybridization as well as bioreactors (Idowu, 2009; Donmez et al., 2016). Bioreactors containing liquid media for micropropagation and rooting started to become more popular thanks to the low production costs (Paek et al., 2001) and the ease of scaling-up (Preil, 1991). Several bioreactor systems have been published (membrane raft system, nutrient mist bioreactor, and temporary immersion bioreactor) (Akita and Takayama, 1994). Among these systems, temporary immersion bioreactor has become more popular. TIS is simple to use and gives mass production rate with minimum physiological disorders (Afreen, 2008).

In practice, TIS wetting the entire culture or plant tissue used as explant with a nutrient medium, and then draining away of the excess nutrient medium under gravity consequently, the plant material has access to air defined as temporary immersion system. TIS usually involves two different cycles; one of them is a wetting, and the other is the drying cycle occurring periodically (Afreen, 2008). Various temporary immersion systems have been developed like RITA, RALM, PLANTIMA, SETIS as well as PlantForm. The RITA system has been found to increase root development in Hevea brasiliensis (Etienne et al., 1997). The bioreactor RALM (Biorreatores RALM, Ralm Industria e Comercio ltda., Brazil) is a TIS, operating on the Twin-Flask principle. The SETIS system (Vervit, Belgium, distributed by Duchefa Biochemie, The Netherlands) operates similarly as the Ebb-and-Flow TIS system. PLANTIMA (A-Tech Bioscientific Co., Ltd., Taiwan) is a small volume TIS, operated on the RITA principle and has been used for plantlet propagation (Yan et al., 2010, 2011). Box-in-Bag is a disposable TIS, operating on the principle of the Ebb-and-Flow TIS. The WAVE bioreactor is a mechanical rocking platform that uses disposable pre-sterilized cultivation bags (Eibl and Eibl, 2006, 2008; Eibl et al., 2009; Georgiev et al., 2014).

An immersion cycle consists of the frequency and duration of immersion. It means immersion should be applied with a planned time and frequency. Immersion cycles directly affect the plant micropropagation by influencing water and nutrient uptake and in addition to that hyperhydricity of the cultured plant tissue (Albarran et al., 2005).

Liquid-based systems have been known to be much more suitable to automation and, consequently, appropriate for the reducing of costs and labor, as a liquid medium could be changed readily during scaling-up, and the cleaning of boxes is simplified compared to agar-based systems (Preil, 1991).

The present study is the first report for myrtle micropropagation with TIS. We aimed to determine the effects of the traditional tissue culture method and PlantForm Temporary Immersion System at in vitro propagation and rooting on Myrtle.

\section{Materials and methods}

\section{Plant material}

Myrtle, Myrtus communis L., genotype was used for in vitro micropropagation and rooting both PlantForm temporary immersion system and traditional solid medium. Plant material was obtained from a commercial seedling company in Adana, Turkey.

\section{Surface sterilization}

Shoot tips of Myrtle plants were cut from donor plants and washed firstly under running tap water about 10 min and then the plant material plunged in $70 \%$ ethanol for 2 min, and dipped in $10 \%$ solution of sodium hypochlorite (commercial bleach solution 
with $4.5 \%$ active chlorine, $\mathrm{v} / \mathrm{v}, \mathrm{NaOCl}$; Domestos $\left.{ }^{\circledR}\right)$ together with $1-2$ drops of Tween20 for 20 min afterward, the plant material was washed three-four times with sterile distilled water.

\section{Multiplication medium}

MS (Murashige and Skoog, 1962) medium were used for multiplication. MS supplemented with $1 \mathrm{mg} \mathrm{l}^{-1}$ BAP (benzylaminopurine), $30 \mathrm{~g} \mathrm{l}^{-1}$ sucrose, $8 \mathrm{~g} \mathrm{l}^{-1}$ agar, $\mathrm{pH}$ 5.8 were prepared and autoclaved at $121^{\circ} \mathrm{C}$ for $15 \mathrm{~min}$. Shoot tips of myrtle were cultured and propagated on MS medium, and incubated in a growth chamber at $25 \pm 2{ }^{\circ} \mathrm{C}$ under cool white fluorescent light at $16 \mathrm{~h}$ photoperiod condition. Plants were propagated until we have enough plant material to experiment with PlantForm system (Fig. 1) and solid medium at the same time.

\section{Micropropagation on PlantForm and solid medium}

MS medium supplemented with $1 \mathrm{mg} \mathrm{l}^{-1} \mathrm{BAP}, 30 \mathrm{~g}^{-1}$ sucrose with $8 \mathrm{~g} \mathrm{l}^{-1}$ agar (all Sigma-Aldrich, St. Louis, USA) were prepared in magentas [( 9 plantlets/magenta) (76.20 x $76.20 \times 10.16 \mathrm{~mm})]$ for solid medium, without agar for PlantForm bioreactor system [120 plantlets/box $(180 \times 150 \times 150 \mathrm{~mm})]$. pH of media was adjusted to 5.8 and autoclaved at $121{ }^{\circ} \mathrm{C}$ for $15 \mathrm{~min}$. The plantlets that developed were subcultured onto fresh MS media in Magenta and PlantForm boxes for three times in every six weeks. Two different immersion time were performed in PlantForm bioreactor system: $15 \mathrm{~min} / 4 \mathrm{~h}$ and $15 \mathrm{~min} / 8 \mathrm{~h}$. The air was renewed for $15 \mathrm{~min} / 4 \mathrm{~h}$ with a pumping system adjusted by an automatic timer for the regulation of aeration and medium supply to the plant material in the temporary immersion system for both treatments. At the end of the three subculture, micropropagation rate, average plant length $(\mathrm{cm})$, number of leaves, fresh weight $(\mathrm{g})$ and dry weight $(\mathrm{g})$ were recorded.

\section{Rooting medium}

Shoots coming from micropropagation were moved to MS medium supplemented with the same components with multiplication medium except for BAP. For rooting 1 $\mathrm{mg} \mathrm{l}^{-1}$ IBA were used. The cultures were maintained incubated in a growth chamber at $25^{\circ} \mathrm{C}$ under cool white fluorescent light at $16 \mathrm{~h}$ photoperiod condition. Six weeks later rooting rate, numbers of roots, fresh weight $(\mathrm{g})$, dry weight $(\mathrm{g})$, length of roots, and plants $(\mathrm{cm})$ were recorded.

The plants were weighed on a precision balance and this was recorded as fresh weight. The plants that fresh weight was determined were weighed after being kept in $70{ }^{\circ} \mathrm{C}$ drying-oven for 3 days and the results were recorded as dry weight. Plant and root lengths were measured with a ruler.
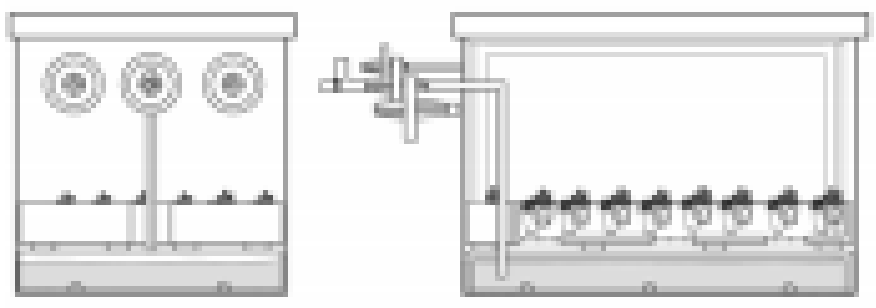

Figure 1. PlantForm TIS bioreactor system (the size is $180 \times 150 \times 150 \mathrm{~mm}$ ) 


\section{Acclimatization}

Plantlets well-developed in PlantForm bioreactor and magenta boxes were removed and roots of the plant material were gently washed under running tap water and plunged in a liquid including $50 \%(\mathrm{w} / \mathrm{v})$ of a $2.5 \mathrm{~g}^{-1}$ fungicide (Captan 50WP, Fruit\&Ornamental, NY, USA) for $10-15 \mathrm{~s}$ and afterward moved to plastic pots $(7 \mathrm{~cm} \times$ $7 \mathrm{~cm}$ width and length) including autoclaved peat (Klasmann, KTS-1) and perlite (1:1, $\mathrm{v} / \mathrm{v})$. Then, myrtle plants were replaced in a controlled greenhouse under natural light at $95-98 \%$ relative humidity and $22-24{ }^{\circ} \mathrm{C}$.

\section{Data analysis}

All studies were performed according to a completely randomized design. Experiments were repeated twice with three replicates. Micropropagation rate was calculated as the number of shoots having after culture/total number of plants. The rooting rate was calculated by dividing the number of rooted plants by the number of total plantlets in rooting assays. All quantitative data calculated as percentage value were subjected to arcsine transformation before variance analysis. All data were expressed as means, and analysis of variance was performed. Means detected the statistically different were separated by least significant difference test (LSD) to evaluate differences among PlantForm $4 \mathrm{~h}-8 \mathrm{~h}$ immersion time and solid medium in Myrtle. Statistical analysis was performed by using JMP ${ }^{\circledR}$ software (SAS Institute, Cary, NC) ver. 8.00.

\section{Results and discussion}

\section{Micropropagation on PlantForm and solid medium}

The effects of PlantForm TIS bioreactor for myrtle were evaluated and compared with the traditional solid medium. Plantlets growing in PlantForm system were better than the solid medium. All data recorded at micropropagation treatments are much more successful. Micropropagation rate $(\%)$, plant length $(\mathrm{cm})$, number of leaves, fresh weight $(\mathrm{g})$ and dry weight $(\mathrm{g})$ coming from PlantForm $4 \mathrm{~h}$ and $8 \mathrm{~h}$ immersion time and solid medium were presented Table 1. Micropropagation rate was detected statistically significant and calculated as 11.40 (PlantForm $8 \mathrm{~h}$ immersion), 8.55 (PlantForm $4 \mathrm{~h}$ immersion), and 6.25 (Solid medium). Similarly, there were important differences in plant length. The average plant length $(\mathrm{cm})$ was detected only $3.12 \mathrm{~cm}$ in solid medium. However, plantlets coming from PlantForm $8 \mathrm{~h}$ immersion time $8.80 \mathrm{~cm}$, PlantForm $4 \mathrm{~h}$ immersion time $7.95 \mathrm{~cm}$. When compared to PlantForm $8 \mathrm{~h}$, PlantForm $4 \mathrm{~h}$ immersion and solid medium, PlantForm systems were effective in plant mass. The mean fresh weight is $1.00,0.59$, and $0.17 \mathrm{~g}$, and dry weight is $0.26,0.11,0.02 \mathrm{~g}$, the number of leaves is 66.70, 51.85, and 38.80, respectively. Apparent differences between the agar medium and PlantForm were shown in Figure 2.

\section{Rooting medium}

The effects of PlantForm TIS bioreactor for rooting in myrtle were evaluated and compared with the traditional solid medium. Plantlets coming from PlantForm and Solid micropropagation media were transferred to rooting media. Rooting rate was calculated as $100 \%$ for PlantForm system in different two immersion time, but in the 
solid medium, only $70 \%$ of plantlets were rooted. The roots were initiated in the media about after 10-15 days of inoculation. All data recorded at rooting treatments are much more successful similar to micropropagation data. Plant length $(\mathrm{cm})$, root length, number of the root, fresh weight $(\mathrm{g})$, and dry weight $(\mathrm{g})$ at PlantForm $4 \mathrm{~h}$ and $8 \mathrm{~h}$ immersion time, and solid medium were presented Table 2. Plant length was calculated the highest in PlantForm $8 \mathrm{~h}$ immersion $(15.10 \mathrm{~cm})$, the lowest in solid medium $(4.59 \mathrm{~cm})$. The results of root length were also similar to plant length. The most successful treatment was detected PlantForm $8 \mathrm{~h}$ immersion time with $8.10 \mathrm{~cm}$. It is only $1.45 \mathrm{~cm}$ in solid medium. It is about 6 times more in PlantForm system than a solid medium. PlantForm system ( $8 \mathrm{~h}$ immersion) was much more effective in root mass when compared to PlantForm $8 \mathrm{~h}$, PlantForm $4 \mathrm{~h}$ immersion time and solid medium. The mean fresh weight is $1.54,0.59$, and $0.44 \mathrm{~g}$, and dry weight is $0.068,0.660$, $0.018 \mathrm{~g}$, the number of roots is $5.40,4.10$, and 2.01 , respectively.

\section{Acclimatization}

Plantlets with shoots and roots from PlantForm bioreactor and magenta boxes were transferred to plastic pots. Plants were successfully acclimatized in a greenhouse. There were no statistical differences among the treatments on survival. However, plants from PlantForm bioreactor systems showed better performance on survival (Fig. 2F).
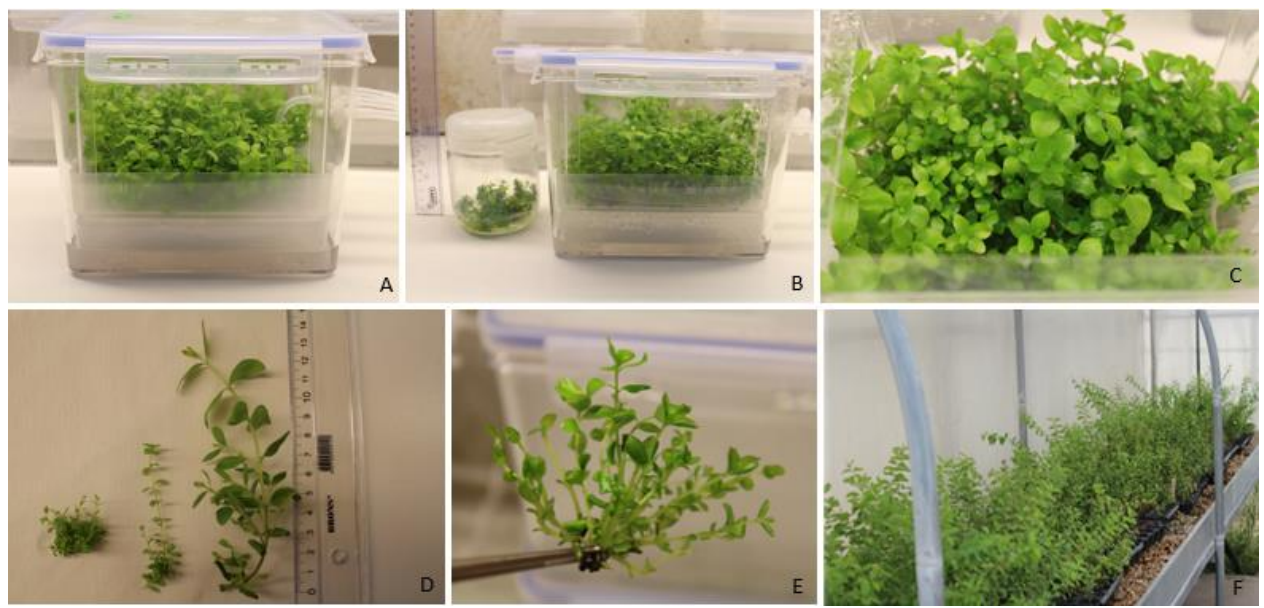

Figure 2. A PlantForm box with myrtle plantlets. B Agar medium and PlantForm. C Plantlets in the PlantForm box. $\boldsymbol{D}$ Myrtle plantlets from PlantForm and agar medium. $\boldsymbol{E}$ A plant from PlantForm. F Acclimatized plants in the greenhouse

Table 1. Data of micropropagation treatments in PlantForm and solid media for myrtle

\begin{tabular}{|c|c|c|c|c|c|}
\hline Medium & $\begin{array}{c}\text { Micropropagation } \\
\text { rate }\end{array}$ & $\begin{array}{c}\text { Plant length } \\
(\mathrm{cm})\end{array}$ & $\begin{array}{c}\begin{array}{c}\text { Number of } \\
\text { leaves }\end{array} \\
\end{array}$ & $\begin{array}{c}\text { Fresh weight } \\
\text { (g) }\end{array}$ & $\begin{array}{c}\text { Dry weight } \\
\text { (g) }\end{array}$ \\
\hline $\begin{array}{l}\text { PlantForm } 8 \mathrm{~h} \\
\text { immersion time }\end{array}$ & $11.40 \mathrm{a}$ & $8.80 \mathrm{a}$ & $66.70 \mathrm{a}$ & $1.00 \mathrm{a}$ & $0.26 \mathrm{a}$ \\
\hline $\begin{array}{c}\text { PlantForm } 4 \mathrm{~h} \\
\text { immersion time }\end{array}$ & $8.55 \mathrm{ab}$ & $7.95 \mathrm{a}$ & $51.85 \mathrm{ab}$ & $0.59 b$ & $0.11 \mathrm{~b}$ \\
\hline Solid medium & $6.25 b$ & $3.12 b$ & $38.80 \mathrm{~b}$ & $0.17 \mathrm{c}$ & $0.02 b$ \\
\hline
\end{tabular}


Table 2. Data on rooting treatments in PlantForm and solid media for myrtle

\begin{tabular}{c|c|c|c|c|c}
\hline Medium & Plant length (cm) & $\begin{array}{c}\text { Number of } \\
\text { roots }\end{array}$ & $\begin{array}{c}\text { Root length } \\
(\mathbf{c m})\end{array}$ & $\begin{array}{c}\text { Fresh weight } \\
(\mathbf{g})\end{array}$ & $\begin{array}{c}\text { Dry weight } \\
(\mathbf{g})\end{array}$ \\
\hline $\begin{array}{c}\text { PlantForm 8 } \mathrm{h} \\
\text { immersion time }\end{array}$ & $15.10 \mathrm{a}$ & $5.40 \mathrm{a}$ & $8.10 \mathrm{a}$ & 1.54 & $0.068 \mathrm{a}$ \\
\hline $\begin{array}{c}\text { PlantForm 4 h } \\
\text { immersion time }\end{array}$ & $11.20 \mathrm{~b}$ & $4.10 \mathrm{a}$ & $3.80 \mathrm{~b}$ & 0.59 & $0.660 \mathrm{a}$ \\
\hline Solid medium & $4.90 \mathrm{c}$ & $2.01 \mathrm{~b}$ & $1.45 \mathrm{c}$ & 0.44 & $0.018 \mathrm{~b}$ \\
\hline
\end{tabular}

$\mathrm{LSD}_{\text {Plantlength }}=1.99, \mathrm{LSD}_{\text {Numberofroot }}=1.66, \mathrm{LSD}_{\text {Rootlength }}=1.75, \mathrm{LSD}_{\text {Freshweight }}=\mathrm{N} . \mathrm{S}, \mathrm{LSD}_{\text {Dryweight }}=0.01$

TIS bioreactors are an alternative in vitro micropropagation mechanism for plant species. There are several different TIS systems; Gravity Immersion Bioreactors (BIG), Temporary Immersion Bioreactors (BIT®), Recipient for Automated Temporary Immersion (RITA $\left.{ }^{\circledR}\right)$. However, these bioreactors are either too small or too heavy to handle with a small interior bottom, which often leads to disorders of cultures due to high density (Welander et al., 2007). In order to solve the problems of TIS, a new culture vessel named PlantForm TIS bioreactor has been recently produced (Welander et al., 2014). Since the first report of a liquid culture in TIS of about 30 years ago, numerous prototypes have been developed. Among these, PlantForm, a recentlydeveloped TIS device, allowing periodic medium immersion and independent ventilation of containers (Lambardi et al., 2015). Immersion frequency could affect micropropagation and rooting in TIS cultures. Therefore, it is a critical issue to adjust optimum immersion time. The effect of immersion frequency $(6,8$ and $12 \mathrm{~h})$ for in vitro propagation of Gerbera jamesonii were investigated by Frómeta et al. (2017) in BIT system. They reported that immersion every $8 \mathrm{~h}$ with additional ventilation produced more shoots with better morphology than the control. However, when shoots were immersed every $6 \mathrm{~h}$, shoots had higher fresh and dry weight. In the present study, we applied two different immersion time $(4 \mathrm{~h}$ and $8 \mathrm{~h}$ ) for myrtle. It was observed the better result in both micropropagation and rooting at $8 \mathrm{~h}$ immersion time. Damiano et al. (2007) investigated that growth performances of Rubus fruticosus, Arbutus unedo and M. communis plants by using a TIS composed of two glass bottles connected with a silicone tube; one of the bottles was used to contain explants which layed on glass beads. Immersion time was adjusted to $120 \mathrm{~min} /$ day. They compared to those in solid and stationary liquid conditions and, determined much higher multiplication rates in the TIS: Rubus fruticosus (x9.3), M. communis (x8.4), Arbutus unedo (x3.6). We also had a higher multiplication rate from PlantForm TIS for myrtle. Here, we detect a better multiplication rate in myrtle; it is high because PlantForm was positively affected the development of plants. Another reason could be the immersion time. Multiplication rate is higher in the $8 \mathrm{~h}$ immersion time than $4 \mathrm{~h}$.

In different plant species, several TIS were used and tested. Ramírez-Mosqueda and Iglesias-Andreu (2016) compared BIT, BIG and RITA systems for multiplication and rooting of Vanilla planifolia. They found that a higher number of shoots/explant were observed in the multiplication phase in BIT systems (18.06 shoots/explant), followed by RITA (12.77) and BIG (6.83). In the rooting phase, a higher number of longer roots were obtained in BIT compared with BIG and RITA. It is clear that different systems could show conflicting results in the same genotype. PlantForm, in our study, gave high 
positive results for micropropagation and rooting on myrtle. The success of PlantForm also was reported by Lambardi et al. (2015). Its performance with ornamental (Carex oshimensis 'Evergreen' and Chrysanthemum morifolium) and fruit species (Ficus carica and Ribes rubrum) is critically analyzed. The results confirmed the convenience of the PlantForm-based TIS for the proliferation of high-quality shoot clusters; moreover, with some species, the system stimulated the contemporary proliferation and rooting of shoots, which can be maintained in proliferation or directly transferred to the acclimatization phase. In another study, the effectiveness of the PlantForm bioreactor in micropropagation of myrtle and olive was examined through measurements of biomass production during the subcultured period in comparison to semi-solid medium culture. The results showed that both species are well adapted to growth in the bioreactor, with survival rates and quality of the crops higher than those obtained under standard culture conditions. Furthermore, the case of myrtle shoot cultures demonstrated the possibility to reduce the concentration of macro and micronutrients in the liquid medium without compromising the growth rates (Benelli et al., 2014).

Temporary immersion bioreactor system could also use for obtaining somatic embryos. Albarran et al. (2005) obtained somatic embryos in Coffea arabica L. using a temporary immersion bioreactor system. They optimized the immersion cycles to increased production and quality of embryos. Researches revealed that increasing the frequency of short immersions ( 1 min immersion every 24, 12 and $4 \mathrm{~h}$ ) affected embryo production (480, 2,094 and 3,081 embryos/1-1 bioreactor, respectively) and improved quality $(60,79$ and $85 \%$ of torpedo-shaped embryos, respectively).

In tissue culture, cytokinins and auxins are remarkable medium contents in detecting the developmental pathways of the plant cells. Cytokinins induce adventitious bud formation in cultures (Rajbhar et al., 2016). Auxins play significant roles in the growth and differentiation of cultured cells and tissues (Alexandrova et al., 1996; Bohidar et al., 2008; Ngomuo et al., 2013). There are some reports that different cytokinins and auxins were used and compared for myrtle micropropagation and rooting in agar media. Rezaee and Kamali (2014) investigated the effect of different media and different plant growth regulations and the concentration of shoot proliferation in myrtle. The results indicated that between, MS and WPM, media, WPM showed better results rather than MS. In shoot proliferation stage were used BA at levels of $0,0.5,1,2,3,4,5$ and $6 \mathrm{mg}$ $\mathrm{I}^{-1}$ and IBA at levels of 0 and $0.1 \mathrm{mg}^{-1}$, then three traits containing: leaf number, shoot length and shoot proliferation were examined. The best result was obtained by using of modified WPM medium with BA in 4 and IBA in $0.1 \mathrm{mg} \mathrm{l}^{-1}$. In the present study, we also used $1 \mathrm{mg} \mathrm{l}^{-1} \mathrm{BAP}$, and we had positive results on the multiplication rate. Different auxins can be used for rooting of plants in in vitro cultures. Auxins such as NAA, IBA, IAA routinely use for plant rooting in vitro cultures. Scarpa et al. (2000) evaluated the influence of two IAA concentrations $\left(0.5 \mathrm{mg} \mathrm{l}^{-1}\right.$ and $\left.1 \mathrm{mg} \mathrm{l}^{-1}\right)$ and different medium to induce root induction of Myrtle. Researchers found that the best rooting results $(61 \%)$ were obtained with 013 medium containing $1 \mathrm{mg} \mathrm{l}^{-1}$ IAA. Hatzilazarou et al. (2001) investigated the rooting capability of two myrtle clones, with large (clone A) and small (clone B) leaves. Shoots transferred to WPM medium containing several concentrations $(0,0.5,1$ or $2 \mu \mathrm{M})$ of IBA, IAA or NAA in rooting assays. According to this study, the best rooting was achieved with the application of $0.5 \mu \mathrm{M}$ IBA (96\% rooting) and $1 \mu \mathrm{M}$ IAA (100\% rooting) for clone A and B, respectively. Ruffoni et al. (2010) determined that IAA and IBA at $0.5 \mathrm{mg} / \mathrm{L}$ increased the rooting percentage and evidenced a difference in the shape and length of the roots in myrtle plant. Aka Kacar et al. (2017) 
investigated the effects of different $\operatorname{IBA}\left(0,1,2,4 \mathrm{mg}^{-1}\right)$ and activated charcoal (AC) $\left(0,0.5,1,2 \mathrm{~g} \mathrm{l}^{-1}\right)$ concentrations on myrtle rooting in in vitro conditions. They used agar MS medium for rooting and reported that the best combination was detected $2 \mathrm{mg} \mathrm{l}^{-1}$ IBA without AC in terms of rooting rate. Similarly, root length, root number, and plant length were recorded less than PlantForm vessels of our study. In the present study, we used IBA for rooting, and it was successful for rooting both in PlantForm and agar media. However, there were apparent differences among the treatments in term of the rooting data. Plants coming from PlantForm were much more at high quality.

\section{Conclusion}

In conclusion, the PlantForm TIS bioreactor represents a valid alternative to conventional systems in vitro micropropagation and rooting, resulting in a reduction of cost, labor, and time for the mass propagation. Comparative investigations between semi-solid medium and bioreactor culture revealed that shoot proliferation and growth were more efficient in the temporary immersion bioreactor system.

Acknowledgments. This research was supported by Çukurova University, Scientific Research Project Unit (Project No: FBA-2015-4865).

\section{REFERENCES}

[1] Afreen, F. (2008): Temporary Immersion Bioreactor. - In: Gupta, S. D., Ibaraki, Y. (eds.) Plant Tissue Culture Engineering. Springer. Netherlands, pp. 187-201.

[2] Aka Kaçar, Y., Şimşek, Ö., Biçen, B., Dal, B. (2017): In vitro rooting of micropropagated shoots from Myrtus communis Linn: influence of activated charcoal and indole-3-butyric acid (IBA). - Acta Horticulture 1155: 531-536.

[3] Akita, M., Takayama, S. (1994): Stimulation of potato (Solanum tuberosum L.) tuberization by semi continuous liquid medium surface level control. - Plant Cell Report 13: 184-187.

[4] Albarran, J., Bertrand, B., Lartaud, M., Etienne, H. (2005): Cycle characteristics in a temporary immersion bioreactor affect regeneration, morphology, water and mineral status of coffee (Coffea arabica) somatic embryos. - Plant Cell, Tissue and Organ Culture 81(1): 27-36.

[5] Alexandrova, K. S., Denchev, P. D., Conger, B. Y. (1996): Micropropagation of switch grass by node culture. - Crop Science 36(6): 1709-1711.

[6] Aydin, C., Özcan, M. M. (2007): Determination of nutritional and physical properties of Myrtle (Myrtus communis L.) fruits growing wild in Turkey. - Journal of Food Engineering 79: 453-458.

[7] Benelli, C., Fernanda, C. M., De Carlo, A. (2015): Plant Form. A Temporary immersion system, for in vitro propagation of $M$. communis and Olea europae. - 6th International Symposium on Production and Establishment of Micropropagated Plants, Sanremo, Italy, Abstract Book.

[8] Bohidar, S., Thirunavoukkarasu, M., Roa, T. V. (2008): Effect of plant growth regulators on in vitro micropropagation of 'Garden Rue' (R. graveolens L.). - International Journal of Integrative Biology 3(1): 36-43.

[9] Casaburi, A., Di Martino, V., Ercolini, D., Parente, E., Villani, F. (2015): Antimicrobial activity of Myrtus communis L. water-ethanol extract against meat spoilage strains of 
Brochothrix thermosphacta and Pseudomonas fragi in vitro and in meat. - Annals of Microbiology 65(2): 841-850.

[10] Dahmoune, F., Nayak, B., Moussi, K., Remini, H., Madani, K. (2015): Optimization of microwave-assisted extraction of polyphenols from Myrtus communis L. leaves. - Food Chemistry 166: 585-595.

[11] Damiano, C., Arias, P. M. D., La Starza, S. R., Frattarelli, A. (2007): Temporary immersion system for temperate fruit trees. - Acta Horticulture 748: 87-90.

[12] Danial, G. H. (2009): Propagation of Myrtus communis L. in vitro. - Journal of Duhok University 12(1): 80-84.

[13] Dönmez, D., Şimşek, Ö., Aka Kaçar, Y. (2016): Genetic engineering techniques in fruit science. - International Journal of Environment, Agriculture and Biotechnology 2(12): $115-128$.

[14] Eibl, R., Eibl, D. (2006): Design and Use of the Wave Bioreactor for Plant Cell Culture. In: Gupta, S. D., Ibaraki, Y. (eds.) Plan Tissue Culture Engineering. Springer, The Netherlands, pp. 203-227.

[15] Eibl, R., Eibl, D. (2008): Design of bioreactors suitable for plant cell and tissue cultures. - Phytochemistry Reviews 7(3): 593-598.

[16] Eibl, R., Werner, S., Eibl, D. (2009): Disposable bioreactors for plant liquid cultures at Litre-scale. - Engineering in Life Sciences 9(3): 156-164.

[17] Etienne, H., Lartaud, M., Michaux-Ferrière, N., Carron, M. P., Berthouly, M., Teisson, C. (1997): Improvement of somatic embryogenesis in Hevea brasiliensis (Müll. Arg) using the temporary immersion techniques. - In Vitro Cellular \& Developmental Biology-Plant 33(2): 81-87.

[18] Frómeta, O. M., Morgado, M. M. E., Da Silva, J. A. T., Morgado, D. T. P., Gradaille, M. A. D. (2017): In vitro propagation of Gerbera jamesonii Bolus ex Hooker f. in a temporary immersion bioreactor. - Plant Cell, Tissue and Organ Culture 129(3): 543-551.

[19] Georgiev, V., Schumann, A., Pavlov, A., Bley, T. (2014): Temporary immersion systems in plant biotechnology. - Engineering in Life Sciences 14(6): 607-621.

[20] Hatzilazarou, S., Grammatikos, H., Economou, A. S., Rifaki, N., Ralli, P. (2001): Rooting in vitro and acclimatization of Myrtus communis microcuttings. - 1st International Symposium on Acclimatization and Establishment of Micropropagated Plants 616: 259-264.

[21] Idowu, P. E., Ibitoye, D. O., Ademoyegun, O. T. (2009): Tissue culture as a plant production technique for horticultural crops. - African Journal of Biotechnology 8(16): 3782-3788.

[22] Lambardi, M., Roncasaglia, R., Bujazha, D., Baileiro, F., Correira Da Silva, D. P., Özüdoğru, E. A. (2015): Improvement of shoot proliferation by liquid culture in temporary immersion. - 6th International Symposium on Production and Establishment of Micropropagated Plants, Sanremo, Italy, Abstract Book.

[23] Murashige, T., Skoog, F. (1962): A revised medium for rapid growth and bio assays with tobacco tissue cultures. - Physiologia Plantarum 15(3): 473-497.

[24] Ngomuo, M., Mneney, E., Ndakidemi, P. (2013): The effects of auxins and cytokinin on growth and development of (Musa sp.) var. "Yangambi" explants in tissue culture. American Journal of Plant Sciences 4(11): 2174-2180.

[25] Paek, K. Y., Hahn. E. J., Son, S. H. (2001): Application of bioreactors for large-scale micropropagation system of plants. - In Vitro Cellular \& Developmental Biology - Plant 37: 149-157.

[26] Preil, W. (1991): Application of Bioreactors in Plant Propagation. - In: Debergh, P. C., Zimmerman, R. H. (eds.) Micropropagation. Kluwer Academic Publishers, Dordrecht, pp. 425-445.

[27] Rajbhar, Y. P., Sumit, T., Hariom, K., Mukesh, K., Anil, K., Govind, R. (2016): Effect of cytokinin and auxin on callus formation and shoot multiplication of strawberry (Fragaria 
$\times$ ananassa Duch.) under in vitro condition. - HortFlora Research Spectrum 5(3): 206212.

[28] Ramírez-Mosqueda, M. A., Iglesias-Andreu, L. G. (2016): Evaluation of different temporary immersion systems $\left(B I T \AA, B I G\right.$, and RITA $\left.{ }^{\circledR}\right)$ in the micropropagation of Vanilla planifolia Jacks. - In Vitro Cellular \& Developmental Biology - Plant 52(2): 154160.

[29] Rezaee, A., Kamali, K. (2014): A New commercial protocol for micropropagation of myrtus tree. - Advances in Bioresearch 5(4): 73-79.

[30] Ruffoni, B., Mascarello, C., Savona, M. (2010): In Vitro Propagation of Ornamental Myrtus (Myrtus Communis). - In: Jain, S. M., Ochatt, S. J. (eds.) Protocols for In Vitro Propagation of Ornamental Plants. Humana Press, Totowa, NJ, pp. 257-269.

[31] San, B., Yildirim, A. N., Polat, M., Yildirim, F. (2015): Chemical compositions of myrtle (Myrtus communis L.) genotypes having bluish-black and yellowish-white fruits. Erwerbs-Obstbau 57(4): 203-210.

[32] Scarpa, G. M., Milia, M., Satta, M. (2000): The influence of growth regulators on proliferation and rooting of in vitro propagated myrtle. - Plant Cell, Tissue and Organ Culture 62(3): 175-179.

[33] Shekafandeh, A. (2007): Effect of different growth regulators and source of carbohydrates on in and ex vitro rooting of Iranian Myrtle. - International Journal of Agricultural Research 2: 152-158.

[34] Sumbul, S., Ahmad, M. A., Asif, M., Akhtar, M. (2011): Myrtus communis Linn. A review. - Indian Journal of Natural Products and Resources 2: 395-402.

[35] Wannes, W. A., Mhamdi, B., Sriti, J., Marzouk, B. (2010): Glycerolipid and fatty acid distribution in pericarp, seed and whole fruit oils of Myrtus communis var. italica. Industrial Crops and Products 31(1): 77-83.

[36] Welander, M., Zhu, L. H., Li, X. Y. (2007): Factors influencing conventional and semiautomated micropropagation. - Propagation of Ornamental Plants 7(3): 103-111.

[37] Welander, M., Persson, J., Asp, H., Zhu, L. H. (2014): Evaluation of a new vessel system based on temporary immersion system for micropropagation. - Scientia Horticulturae 179: 227-232.

[38] Yan, H., Liang, C., Li, Y. (2010): Improved growth and quality of Siraitia grosvenorii plantlets using a temporary immersion system. - Plant Cell, Tissue and Organ Culture (PCTOC) 103(1): 131-135.

[39] Yan, H., Yang, L., Li, Y. (2011): Improved growth and quality of Dioscorea fordii Prain et Burk and Dioscorea alata plantlets using a temporary immersion system. - African Journal of Biotechnology 10(83): 19444-19448. 